\title{
Real-time Detection of Pace Pulses in a Single Lead ECG
}

\author{
Irena Jekova ${ }^{1}$, Serafim Tabakov², Ivo Iliev², Valentin Tsibulko², Krasimira Kostikova ${ }^{3}$ \\ ${ }^{1}$ Institute of Biophysics and Biomedical Engineering, Bulgarian Academy of Sciences, Sofia, Bulgaria \\ ${ }^{2}$ Technical University, Sofia, Bulgaria \\ ${ }^{3}$ Medical University, Sofia, Bulgaria
}

\begin{abstract}
This paper presents an algorithm for real-time pace pulses detection in a single-lead ECG, based on assessment of the cumulative slope of the ECG signal calculated in a narrow time window. The algorithm is trained and tested on an artificial database containing 780 ECGs in lead II (390 for training, 390 for testing) that represent different arrhythmias, combined with artificially superimposed pace pulses, which cover the wide ranges of rising edge $(<10 \mu \mathrm{s}$ to $100 \mu \mathrm{s})$ and total pulse durations (100 $\mu \mathrm{s}$ to $2 \mathrm{~ms}$ ) and correspond to various pacemaker modes. The achieved accuracy is $\mathrm{Se}=99.3 \%, P P V=99.0 \%$ for ECGs sampled at $F s=32 \mathrm{kHz}$, and $\mathrm{Se}=97.1 \%, P P V=96.8 \%$ for $\mathrm{Fs}=16 \mathrm{kHz}$.
\end{abstract}

\section{Introduction}

Correct detection of pacemaker pulses in the electrocardiogram (ECG) is crucial for proper evaluation of its functionality and its effect on the cardiac rhythm, as well as for elimination of the pace pulses influence on the automatic ECG analysis.

The pace pulse is bipolar, with fast rising edge about $10 \mu$ s; amplitude on the patient skin surface varying between few hundred $\mu \mathrm{V}$ to several hundred $\mathrm{mV}$; and width of pace artifacts between $100 \mu$ s and $2 \mathrm{~ms}$ [1]. Depending on the number of active leads the pacemakers are single chamber, dual chamber and bi-ventricular, while according to their programming the devices could be with fixed-rate, on demand and rate-responsitive [2]. This inevitably leads to a number of challenges for the design of algorithms for pace pulses detection.

There are different medical standards with variable requirements regarding the height and width of the pace pulse that has to be captured and indicated on the screen of the device. According to ANSI/AAMI EC11 [3] the features of the pacemaker pulses that should be obligatory detected are as follows: (i) duration from $100 \mu$ s to $2 \mathrm{~ms}$; (ii) amplitude from $2 \mathrm{mV}$ to $250 \mathrm{mV}$; (iii) frequency up to 100 pulses per minute; (iv) rising edge duration less than
100 ms. The IEC60601-2-27 standard [4] states duration from $0.5 \mathrm{~ms}$ to $2.0 \mathrm{~ms}$ and amplitude from $2 \mathrm{mV}$ to 700 $\mathrm{mV}$. Modern pacemakers could generate smaller pace pulse amplitudes that could fall below the requirements set in the standards and lead to complications in the algorithms for pace pulses detection [2].

Publicly presented pace pulse detection methods rely on hardware decisions [5] and software analysis after specific filtration [6,7] and multi-lead ECG processing [1]. The digital ECG analysis should be applied on highresolution ECG $[8,9]$ that preserves the frequency content of the pacing pulses.

This paper presents an algorithm for real-time pace pulses detection in a single ECG lead.

\section{Database}

The ECG signals used for training and testing are taken from an artificial database containing ECG recordings in lead II that represent different arrhythmias generated by HKP (Heidelberger Praxisklinik) simulator, combined with artificially superimposed pace pulses that cover the wide ranges of rising edge ( $<10 \mu \mathrm{s}$ to $100 \mu \mathrm{s})$ and total pulse durations (100 $\mu \mathrm{s}$ to $2 \mathrm{~ms}$ ) and correspond to various pacemaker modes [10]. The database comprises 780 'pure' ECGs with pace pulses with duration of $10 \mathrm{~s}$ and annotated positions of the pace pulses. The signals are recorded with $9.81 \mu \mathrm{V} / \mathrm{LSB}$ amplitude resolution at $128 \mathrm{kHz}$ sampling rate, which preserves the steep raising and trailing edges of the pace pulses. The algorithm was trained on a set of 390 randomly selected recordings and tested on the remaining 390 ECGs.

\section{Method}

The algorithm for pace pulses detection is based on assessment of the cumulative slope of the ECG signal (Slope) calculated in a narrow time window ( $N$ samples), according to the equation:

$$
\operatorname{Slope}(j)=\left(2 * N * E C G(j)-\sum_{i=j-N}^{j+N} E C G(i)\right)^{2}
$$

Block-diagram of the algorithm is presented in Fig. 1. 
Slope(j) is compared to a falling threshold SlopeTHR with predefined initial (SlopeTHRInit) and minimal (SlopeTHRMin) values. When Slope(j) exceeds SlopeTHR a pace pulse is detected, SlopeTHR is updated and the detection of pace pulses is disabled for the next $10 \mathrm{~ms}$ in order to prevent consequent detections of the same pace pulse. After the detection procedure is unblocked, SlopeTHR is slowly decreased with coefficient $K$ until new pace pulse is detected or SlopeTHRMin is reached.

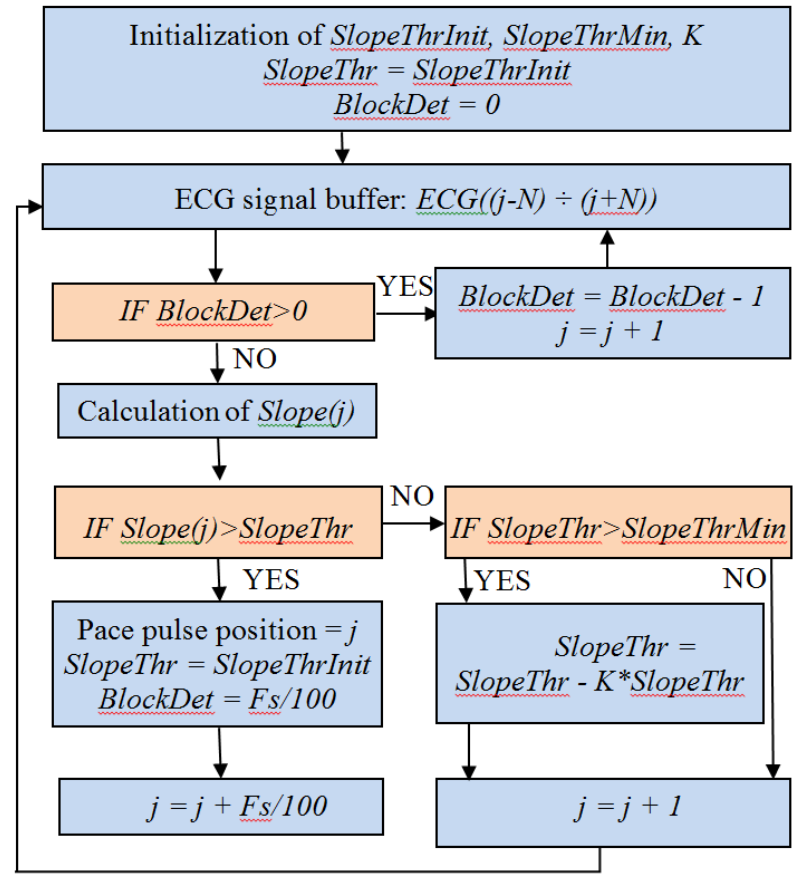

Figure 1. Block-diagram of the pace pulses detection algorithm.

We used the ECG recordings from the training dataset to assess the influence of the adjustable parameters $N$, SlopeThrInit, SlopeThrMin, $K$ and the sampling frequency $(F s)$ on the reliability of pace pulses detection, and to select values, which could provide optimal detection accuracy.

\subsection{Adjustment of $N$ and SlopeThr}

Two auxiliary measures, representing the maximal Slope value within $10 \mathrm{~ms}$ around the pace pulses annotations (MaxInPMann) and out of these intervals (MaxOutPMann), were calculated for ten different values of the time window $\mathrm{N}(\mathrm{N}=0.1 \mathrm{~ms}, 0.25 \mathrm{~ms}, 0.5 \mathrm{~ms}$, $0.75 \mathrm{~ms}, 1 \mathrm{~ms}, 1.5 \mathrm{~ms}, 2 \mathrm{~ms}$, 3ms, $4 \mathrm{~ms}$, $5 \mathrm{~ms}$ ), as follows:

- $\operatorname{MaxInPMann}(i)=\max (\operatorname{Slope}(\operatorname{ANN}(i) \pm 10 \mathrm{~ms}))$

- MaxOutPMann(i) $=\max (\operatorname{Slope}(\operatorname{ANN}(\mathrm{i}-1)+10 \mathrm{~ms}$ to ANN(i)-10ms))

where $i$ is the consecutive number of the pace pulse. Based on MaxInPMann, MaxOutPMann values for each couple ( $N$, SlopeThr) two statistical indices are defined:
- Sensitivity for detection of pace pulses:

Se $=100 * \operatorname{count}($ MaxInPMann $\geq$ SlopeThr $) / \operatorname{count}(A N N)$

- Specificity for detection of pace pulses:

$S p=100 *$ count $($ MaxOutPMann $<$ SlopeThr $) /$ count $($ ANN $)$

Receiver-operating characteristics (ROC) curves are used for selection of the optimal couple ( $N$, SlopeThr).

\subsection{Investigation of the influence of $F s$}

To assess the influence of $F s$ on the pace detection accuracy we calculated Se, Sp for the defined optimal time window $N$, different values of SlopeThr and $F s=4$, 8, 16, 32, 64, $128 \mathrm{kHz}$. The maximal detection accuracy for each Fs was considered.

\subsection{Selection of SlopeThrInit, SlopeThrMin and adjustment of $K$}

The ranges for adjustment of SlopeThrInit and SlopeThrMin were selected by considering the accuracy results for different fixed thresholds. Six $K$ values were tested $-K(\%)=1,1 / 2,1 / 4,1 / 8,1 / 16,1 / 32$, i.e. $K(\%$ o $)=$ $10,5,2.5,1.25,0.625,0.3125$.

The accuracy for the different combinations between SlopeThrInit, SlopeThrMin and $K$ was estimated via:

- Sensitivity: $S e=100 * T P / T P+F N$

- Positive predictive value: $P P V=100 * T P /(T P+F P)$, where $T P$ (true positive) is the number of the correctly detected pace pulses; $F N$ (false negative) is the number of not detected pace pulses; FP (false positive) is the number of the erroneously detected pace pulses.

\section{Results and discussion}

The results achieved during the adjustment of the time window $N$ and the optimal fixed value of the threshold SlopeThr for selected $\mathrm{Fs}=32 \mathrm{kHz}$ are presented via the ROC curves in Fig. 2. The ROC curves analysis highlights the following settings:

- $\quad N=1 \mathrm{~ms}$, SlopeThr=1.28V; $\mathrm{Sp}=100 \%$; $\mathrm{Se}=99.28 \%$;

- $\quad N=1.5 \mathrm{~ms}$, SlopeThr=3.84V; Sp=100\%; Se=98.67\%;

- $\quad N=1.5 \mathrm{~ms}$, SlopeThr=2.35V; $\mathrm{Sp}=99.97 \% ; \mathrm{Se}=99.52 \%$;

- $\quad N=1.5 \mathrm{~ms}$, SlopeThr=2.16V; Sp=97.04\%; Se=100\%;

Based on the ROC curves in Fig. 2 a time window $N=1.5 \mathrm{~ms}$ (providing balanced Se, Sp) is selected and the influence of $F s$ on the detection accuracy is investigated. The results are illustrated in Fig. 3. The ascending trend of the mean(Se,Sp) exceeds $99.5 \%$ for all $F s \geq 32 \mathrm{kHz}$. Mean(Se,Sp) falls down to $98 \%$ for $F s=16 \mathrm{kHz} ; 95.5 \%$ for $F s=8 \mathrm{kHz}$ and $76.5 \%$ for $F s=4 \mathrm{kHz}$. Obviously, the choice of sampling frequency should be a compromise between detection accuracy on one side and the necessary processing resources and memory space for real-time analysis on the other. 


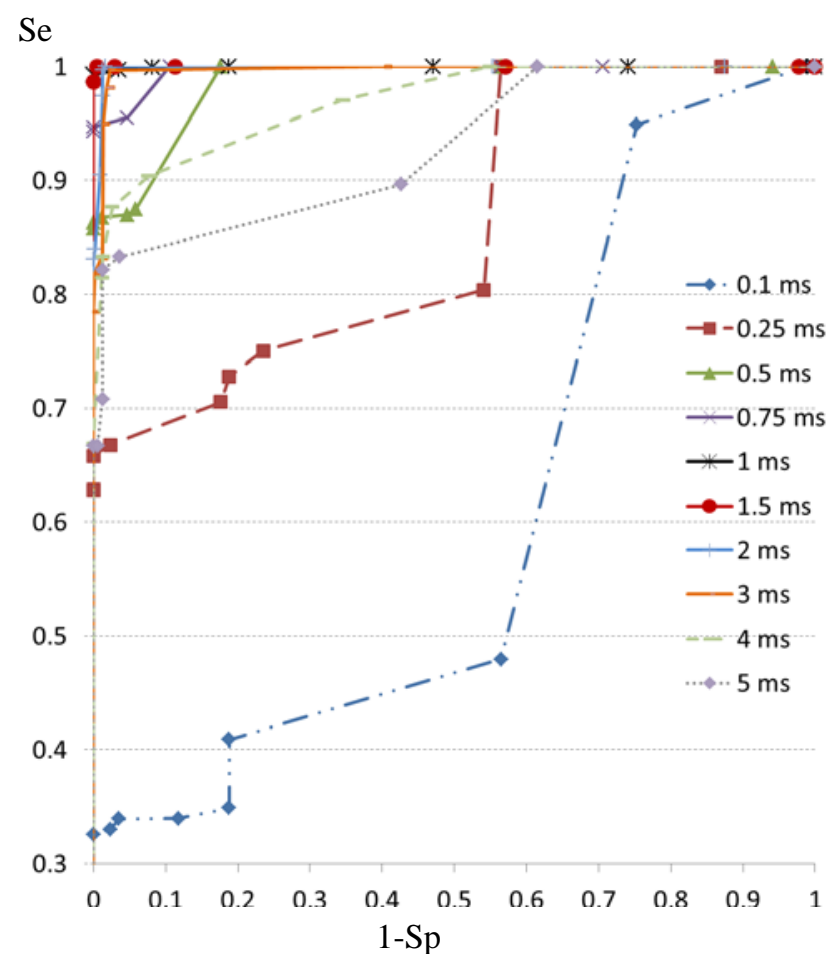

Figure 2. ROC curves tor altterent windows $N$ and SlopeThr.

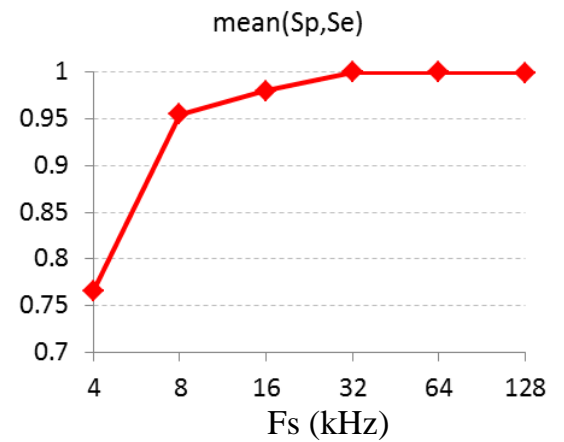

Figure 3. Influence of Fs on the detection accuracy.

Table 1. Accuracy of pace pulses detection for different Fs and SlopeThr

\begin{tabular}{cccc}
\hline Fs (kHz) & SlopeThr (V) & Sp (\%) & Se (\%) \\
\hline \multirow{3}{*}{$32 \mathrm{kHz}$} & 3.82 & 100 & 98.67 \\
& 2.35 & 99.52 & 99.97 \\
& 2.16 & 97.04 & 100 \\
\hline \multirow{3}{*}{$16 \mathrm{kHz}$} & 1.32 & 100 & 96.26 \\
& 1.27 & 98.82 & 96.53 \\
& 1.18 & 96.41 & 96.92 \\
& 1.08 & 94.03 & 97.44 \\
& 0.98 & 86.88 & 97.98 \\
& 0.78 & 80.81 & 99.43 \\
& 0.59 & 79.34 & 100 \\
\hline
\end{tabular}

Adjustment of initial threshold (SlopeThrInit), minimal threshold (SlopeThrMin) and coefficient $K$ for $N=1.5 \mathrm{~ms}$, $F s=32 \mathrm{kHz}$ and $F s=16 \mathrm{kHz}$ is based on the accuracy results obtained for stationary threshold (Table 1).
Adjustments for Fs=32 kHz: We select SlopeThrMin = $2.16 \mathrm{~V}$, for which $\mathrm{Se}=100 \%$. Lower values of SlopeThrMin would only decrease Sp without any improvement of Se. Different $\mathrm{K}$ values are studied for SlopeThrInit $=3.82 \mathrm{~V}$ and SlopeThrInit $=2.35 \mathrm{~V}$. The results are illustrated in Fig. 4a,b. The optimal combination of parameters for $\mathrm{Fs}=32 \mathrm{kHz}$ is SlopeThrInit $=3.82 \mathrm{~V}$, SlopeThrMin $=2.16 \mathrm{~V}, \mathrm{~K}=0.3125 \%$ with detection accuracy $\mathrm{Se}=99.24 \%$, $\mathrm{PPV}=98.95 \%$.

Adjustments for Fs=16 kHz: We select SlopeThrMin = $1.08 \mathrm{~V}$, for which $\mathrm{Se}=97.44 \%, \mathrm{Sp}=94.03 \%$. Lower values of SlopeThrMin would lead to insignificant increase of Se at the cost of considerable decrease in Sp. SlopeThrInit is set to $1.32 \mathrm{~V}$, for which $\mathrm{Sp}=100 \%$, and different $\mathrm{K}$ values are tested. The results are illustrated in Fig. 4c. Depending on the particular application and the preset requirements, for $\mathrm{Fs}=16 \mathrm{kHz}$ one could select among (i) setting for maximal Se (Se=97.09\%, PPV=96.75\%) SlopeThrInit $=1.32 \mathrm{~V}$, SlopeThrMin $=1.08 \mathrm{~V}$, $\mathrm{K}=1.25 \%$; (ii) setting for maximal $\mathrm{PPV}(\mathrm{Se}=96.25 \%$, PPV=97.86\%) - SlopeThrInit $=1.32 \mathrm{~V}$, SlopeThrMin $=$ $1.08 \mathrm{~V}, \mathrm{~K}=0 \%$.

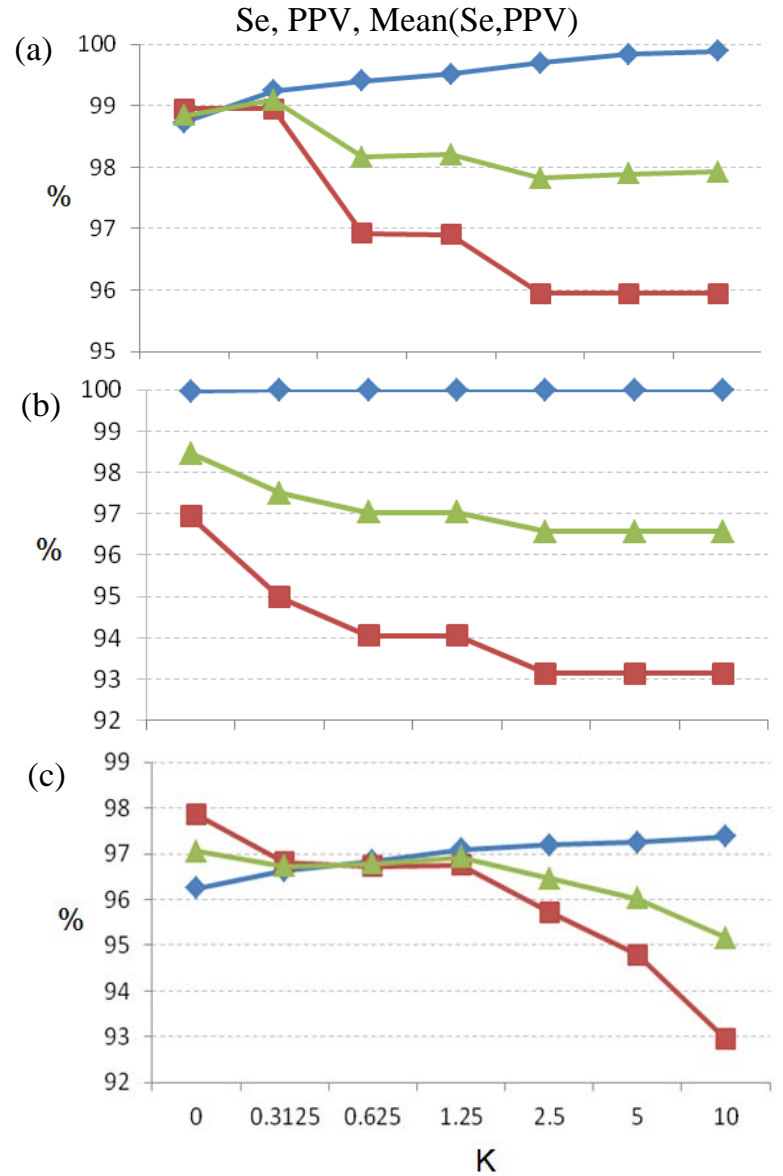

Fig. 4. Relation between Se (blue), PPV (red), mean(Se,PPV) (green) and the coefficient $\mathrm{K}$ for: (a) SlopeThrInit $=3.82 \mathrm{~V}, \mathrm{Fs}=32 \mathrm{kHz}$; (b) SlopeThrInit $=2.35 \mathrm{~V}$, Fs=32kHz; (c) SlopeThrInit $=1.32$ V, Fs $=16 \mathrm{kHz}$. 
The developed algorithm for pace pulses detection is tested in Matlab environment with the following settings:

- Setting 1: Fs $=32 \mathrm{kHz}$, SlopeThrInit $=3.82 \mathrm{~V}$, SlopeThrMin $=2.35 \mathrm{~V}, \mathrm{~K}=0.3125 \%$;

- Setting 2: Fs $=16 \mathrm{kHz}$, SlopeThrInit $=1.32 \mathrm{~V}$, SlopeThrMin $=1.08 \mathrm{~V}, \mathrm{~K}=0 \%$;

- Setting 3: Fs $=16 \mathrm{kHz}$, SlopeThrInit $=1.32 \mathrm{~V}$, SlopeThrMin $=1.08 \mathrm{~V}, \mathrm{~K}=1.25 \%$.

The test results are presented in Table 2 .

Table 2. Accuracy of pace pulses detection over the test dataset, calculated for different settings.

\begin{tabular}{rrr}
\hline Settings & \multicolumn{1}{c}{ Se (\%) } & PPV (\%) \\
\hline Setting 1 & 99.27 & 98.95 \\
Setting 2 & 96.14 & 97.85 \\
Setting 3 & 97.14 & 96.75 \\
\hline
\end{tabular}

The operation of the developed algorithm with Setting 3 $(\mathrm{Fs}=16 \mathrm{kHz}$, SlopeThrInit $=1.32 \mathrm{~V}$, SlopeThrMin $=1.08$ $\mathrm{V}, \mathrm{K}=1.25 \%$ ) is illustrated via the examples in figures 5 and 6. The false negative errors (decreasing Se) are due mainly to signals with low-amplitude pace pulses, while the false positive errors (decreasing Sp) result from steep and high-amplitude ectopic beats.
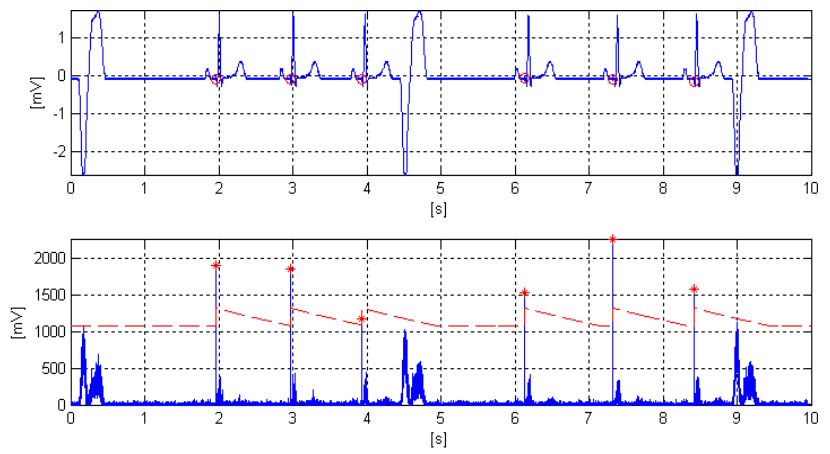

Fig. 5. Correct detection of low-amplitude pace pulses (red ' 0 ' in $1^{\text {st }}$ subplot mark the annotations, red ' $*$ ' in $2^{\text {nd }}$ subplot show the detections). The relatively high cumulative slope under the steep and high-amplitude ectopic beats does not lead to false pace pulses detection.
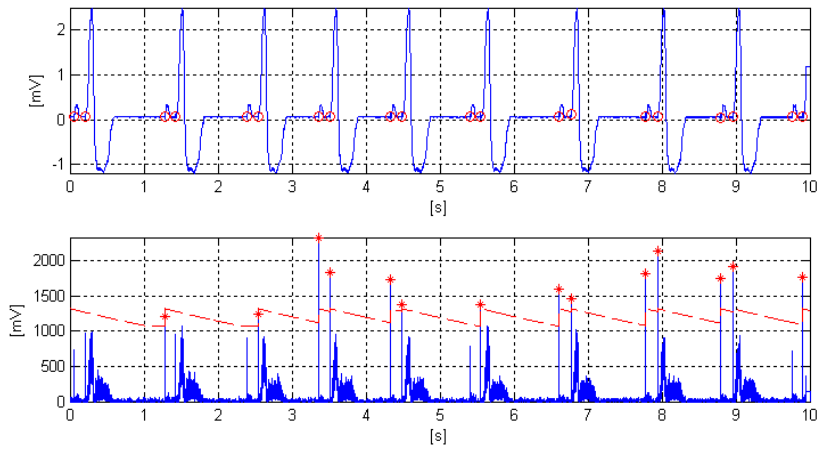

Fig. 6. Low-amplitude pace pulses not detected by the algorithm due to the low cumulative slope, which is comparable to the one for the ventricular complexes.

\section{Conclusions}

This study addresses the development and testing of an algorithm for detection of pace pulses based on quasireal-time assessment of the cumulative ECG slope (delay of $1.5 \mathrm{~ms}$ ). The designed method performs reliably and guarantees $\mathrm{Se}=99.3 \%$ and $\mathrm{PPV}=99.0 \%$ for single lead ECG sampled at Fs $=32 \mathrm{kHz}$. Expectedly, when Fs is decreased to $16 \mathrm{kHz}$ the detection accuracy drops down with $2 \%$ (Se=97.1\%, PPV=96.8\%), generally due to false negative errors occurring for low-amplitude pace pulses.

\section{Acknowledgements}

This study is supported by the Bulgarian Scientific Research Fund, grant ДН17/19.

\section{References}

[1] Kruse J., Redmond C. Detecting and distinguishing cardiac pacing artifacts. Analog Dialogue 2012;46:13-8.

[2] Tsibulko V, Iliev I, Jekova I. A review on pacemakers: Device types, operating modes and pacing pulses. Problems related to the pacing pulses detection. Int. J. Bioautomation 2014;18(2):89-100.

[3] American National Standard. Diagnostic electrocardiographic devices, ANSI/AAMI EC11, 2007.

[4] American National Standard. Medical electrical equipment - Part 2-27: Particular requirements for the basic safety and essential performance of electrocardiographic monitoring equipment, ANSI/AAMI/IEC 60601-2-27, 2011

[5] Calabria T. Hardware pace using slope detection. Texas Instruments Precision Designs: Verified Design, 2007.

[6] Herleikson E. ECG pace pulse detection and processing. US5682902 A04-Nov-1997.

[7] Polpetta A, Banelli P. Fully digital pacemaker detection in ECG signals using a non-linear filtering approach. Proc. 30th Annual International Conference of the IEEE Engineering in Medicine and Biology Society, EMBS'2008 2008:5406-10.

[8] Petrutiu S, Sahakian A, Ricke A, Young B, Swiryn S. High resolution electrocardiography optimized for recording pulses from electronic pacemakers: Evaluation of a new pacemaker sensing system. Computers in Cardiology 2007;34:197-200.

[9] Luo S, Johnston P, Hong W. Performance study of digital pacer spike detection as sampling rate changes. Computing in Cardiology 2008;35:349-52.

[10] Jekova I, Tsibulko V, Iliev I. ECG Database Applicable for Development and Testing of Pace Detection Algorithms. Int. J. Bioautomation 2014;18(4):377-88.

Address for correspondence.

Irena Jekova

Bulgarian Academy of Sciences,

Institute of Biophysics and Biomedical Engineering, Acad. G. Bonchev str., bl.105, 1113, Sofia, Bulgaria irena@biomed.bas.bg. 